\title{
THE SYSTEM OF GENERALIZED SET-VALUED EQUILIBRIUM PROBLEMS
}

\author{
JIAN-WEN PENG
}

Received 14 September 2004; Revised 11 November 2004; Accepted 18 November 2004

We introduce new and interesting model of system of generalized set-valued equilibrium problems which generalizes and unifies the system of set-valued equilibrium problems, the system of generalized implicit vector variational inequalities, the system of generalized vector and vector-like variational inequalities introduced by Ansari et al. (2002), the system of generalized vector variational inequalities presented by Allevi et al. (2001), the system of vector equilibrium problems and the system of vector variational inequalities given by Ansari et al. (2000), the system of scalar variational inequalities presented by Ansari Yao (1999, 2000), Bianchi (1993), Cohen and Caplis (1988), Konnov (2001), and Pang (1985), the system of Ky-Fan variational inequalities proposed bt Deguire et al. (1999) as well as a variety of equilibrium problems in the literature. Several existence results of a solution for the system of generalized set-valued equilibrium problems will be shown.

Copyright (c) 2006 Jian-Wen Peng. This is an open access article distributed under the Creative Commons Attribution License, which permits unrestricted use, distribution, and reproduction in any medium, provided the original work is properly cited.

\section{Introduction}

Throughout this paper, let $I$ be an index set. For each $i \in I$, let $Z_{i}$ be a topological vector space. $C_{i}: X \rightarrow 2^{Z_{i}}$ be a set-valued mapping such that $C_{i}(x)$ is a closed pointed and convex cone with $\operatorname{int} C_{i}(x) \neq \varnothing$ for each $x \in X$, where int $A$ denotes the interior of the set $A$. For each $i \in I$, let $E_{i}$ and $F_{i}$ be two locally convex Hausdorff topological vector spaces. Consider two family of nonempty compact convex subsets $\left\{X_{i}\right\}_{i \in I}$ and $\left\{Y_{i}\right\}_{i \in I}$ with $X_{i} \subset$ $E_{i}$ and $Y_{i} \subset F_{i}$. Let $E=\prod_{i \in I} E_{i}, X=\prod_{i \in I} X_{i}, F=\prod_{i \in I} F_{i}$ and $Y=\prod_{i \in I} Y_{i}$. An element of the set $X^{i}=\prod_{j \in I \backslash i} X_{i}$ will be denoted by $x^{i}$, therefore, $x \in X$ will be written as $x=$ $\left(x^{i}, x_{i}\right) \in X^{i} \times X_{i}$. An element of the set $Y$ will be denoted by $y=\prod_{i \in I} y_{i}$, where $y_{i}$ is an element of the set $Y_{i}$. Let $T_{i}: X \rightarrow 2^{Y_{i}}$ and $\Psi_{i}: X \times Y_{i} \times X_{i} \rightarrow 2^{Z_{i}}$ be set-valued mappings.

The system of generalized set-valued equilibrium problems (in short, SGSEP) which is a family of generalized set-valued equilibrium problems defined on a product set will be introduced as follows: The (SGSEP) is to find $\left(\bar{x}, \overline{y_{i}}\right)$ in $X \times Y_{i}$ such that for each $i \in I$,

Hindawi Publishing Corporation Journal of Inequalities and Applications Volume 2006, Article ID 16764, Pages 1-9 DOI 10.1155/JIA/2006/16764 
2 The system of generalized set-valued equilibrium problems

$\overline{x_{i}} \in X_{i}, \overline{y_{i}} \in T_{i}(\bar{x})$ and

$$
\Psi_{i}\left(\bar{x}, \overline{y_{i}}, z_{i}\right) \not \subset-\operatorname{int} C_{i}(\bar{x}), \quad \forall z_{i} \in X_{i}
$$

The (SGSEP) is a new, interesting, meaningful and general mathematical model, which contains many mathematical models as special cases, for some examples.

(i) For each $i \in I$, if $Y_{i}=\left\{\bar{y}_{i}\right\}$ and $T_{i}(x)=\left\{\overline{y_{i}}\right\}$ for all $x \in X$, define a function $\Phi_{i}: X \times$ $X_{i} \rightarrow Z_{i}$ as $\Phi_{i}\left(x, z_{i}\right)=F_{i}\left(x, \overline{y_{i}}, z_{i}\right)$, then the (SGSEP) reduces to the system of set-valued equilibrium problems (in short, SSEP), which is to find $\bar{x}$ in $X$ such that for each $i \in I$,

$$
\Phi_{i}\left(\bar{x}, z_{i}\right) \not \subset-\operatorname{int} C_{i}(\bar{x}), \quad \forall z_{i} \in X_{i}
$$

(ii) For each $i \in I$, let $L\left(E_{i}, F_{i}\right)$ denote the continuous linear operators from $E_{i}$ to $F_{i}$, and $V_{i}: X \rightarrow 2^{L\left(E_{i}, F_{i}\right)}$ be a set-valued mapping, let $\psi_{i}: L\left(E_{i}, F_{i}\right) \times X_{i} \times X_{i} \rightarrow Z_{i}$ be a vectorvalued mapping. Then a special case of the (SSEP) is the system of generalized implicit vector variational inequalities (in short, SGIVVI), which is to find $\bar{x} \in X$ such that for each $i \in I$,

$$
\forall y_{i} \in X_{i}, \quad \exists \overline{u_{i}} \in V_{i}(\bar{x}): \psi_{i}\left(\overline{u_{i}}, \overline{x_{i}}, y_{i}\right) \notin-\operatorname{int} C_{i}(\bar{x}) .
$$

(iii) For each $i \in I$, let $\eta_{i}: X_{i} \times X_{i} \rightarrow E_{i}$ be a bifunction, then a special case of the (SGIVVI) is the system of generalized vector variational-like inequalities (in short, SGVVLI), which is to find $\bar{x} \in X$ such that for each $i \in I, \forall y_{i} \in X_{i}, \exists \overline{u_{i}} \in V_{i}(\bar{x})$ : $\left\langle\overline{u_{i}}, \eta_{i}\left(y_{i}, \overline{x_{i}}\right)\right\rangle \notin-\operatorname{int} C_{i}(\bar{x})$.

(iv) A special case of the (SGVVLI) is the system of generalized vector variational inequalities (in short, SGVVI), which is to find $\bar{x} \in X$ such that for each $i \in I$,

$$
\forall y_{i} \in X_{i}, \quad \exists \overline{u_{i}} \in V_{i}(\bar{x}):\left\langle\overline{u_{i}}, y_{i}-\overline{x_{i}}\right\rangle \notin-\operatorname{int} C_{i}(\bar{x})
$$

and if $C_{i}(x)=C$ for all $x \in X$, then the (SGVVI) becomes the problem considered by Allevi et al. in [1] with relative pseudomonotonicity. If $V_{i}$ is a single-valued function and $C_{i}(x)=C$ for all $x \in X$, then the (SGVVI) reduces to the system of vector variational inequalities (in short, SVVI), which is to find $\bar{x} \in X$ such that for each $i \in I$,

$$
\left\langle V_{i}(\bar{x}), y_{i}-\overline{x_{i}}\right\rangle \notin-\operatorname{int} C, \quad \forall y_{i} \in X_{i}
$$

Moreover, if $Z_{i}=R$ and $C=R^{+}=\{r \in R: r \geq 0\}$, then the (SVVI) reduces to the system of (scalar) variational inequalities (in short, SVI), which is to find $\bar{x} \in X$ such that for each $i \in I$,

$$
\left\langle V_{i}(\bar{x}), y_{i}-\overline{x_{i}}\right\rangle \geq 0, \quad \forall y_{i} \in X_{i}
$$

The (SVI) was studied by Pang [27], Cohen and Chaplais [15], Ansari and Yao [5, 7], Bianchi [9] and Konnov [21]. 
(v) For each $i \in I$, if $\Phi_{i}$ is replaced by a single-valued mapping $\varphi_{i}: X \times X_{i} \rightarrow Z_{i}$, then the (SSEP) reduces to a system of vector equilibrium problems (in short, SVEP), which is to find $\bar{x}$ in $X$ such that for each $i \in I$,

$$
\varphi_{i}\left(\bar{x}, z_{i}\right) \notin-\operatorname{int} C_{i}(\bar{x}), \quad \forall z_{i} \in X_{i}
$$

For each $i \in I$, let $f_{i}: X \rightarrow Z_{i}$ be a vector-valued function. If for each $i \in I$,

$$
\varphi_{i}\left(x, z_{i}\right)=f_{i}\left(x^{i}, z_{i}\right)-f_{i}(x)
$$

then the (SVEP) is equivalent to the generalized Nash equilibrium problem (in short, GNEP), which is to find $\bar{x} \in X$ such that for each $i \in I, f_{i}\left(\overline{x^{i}}, z_{i}\right)-f_{i}(\bar{x}) \notin-\operatorname{int} C(\bar{x}), \forall z_{i} \in$ $X_{i}$.

The (SSEP), the (SGIVVI), the (SGVVLI), the (SGVVI), the (SVEP) and the (GNEP) were introduced and studied by Ansari et al. in [4].

If $Z_{i}=Z, C_{i}(x)=C$ for each $i \in I$ and for all $x \in X$, then the (SVEP) becomes the problem studied by Ansari et al. in [3] and contains the system of vector optimization problems, the Nash equilibrium problem for vector-valued functions and the (SVVI) as special cases.

If $Z_{i}=R, C_{i}(x)=\{r \in R: r \leq \lambda\}$ for each $i \in I$ and for all $x \in X$, then the (SVEP) reduces the system of (scalar) Ky-Fan variational inequalities which is to find $\bar{x} \in X$ such that for each $i \in I$,

$$
\varphi_{i}\left(\bar{x}, z_{i}\right) \leq \lambda, \quad \forall z_{i} \in X_{i}
$$

This problem was studied by Deguire et al. [16].

(vi) If $I=1$, then the (SGSEP) reduces to the generalized set-valued equilibrium problem (in short, GSEP), which is to find $\bar{x} \in X$ and $\bar{y} \in T(\bar{x})$ such that

$$
\Psi(\bar{x}, \bar{y}, z) \not \subset-\operatorname{int} C(\bar{x}), \quad \forall z \in X
$$

This problem was introduced and studied by $\mathrm{Fu}$ and Wan [18].

If $I=1$, then the (SSEP) reduces to the set-valued equilibrium problem (in short, SEP), which is to find $\bar{x} \in X$ such that

$$
\Phi(\bar{x}, z) \not \subset-\operatorname{int} C(\bar{x}), \quad \forall z \in X
$$

The (SEP) was studied by Ansari et al. [2], Ansari and Yao [6], Konnov and Yao [22], Lin et al. [23], Oettli and Schlager [26], and the (SEP) contains the vector equilibrium problem in $[10,13,19,23,25,28]$ and the equilibrium problem in $[11,12]$ as special cases.

In this paper, some existence results of a solution for the (SGSEP) will be shown. These results improve and generalize the main results in $[3,4]$. 
4 The system of generalized set-valued equilibrium problems

\section{Basic definitions}

In order to prove the main results, it is need to introduce the following new definitions.

Definition 2.1. Let $C_{i}: X \rightarrow 2^{Z_{i}}$ be a set-valued mapping such that $C_{i}(x)$ is a closed pointed and convex cone with int $C_{i}(x) \neq \varnothing$ for each $x \in X$. Then the set-valued mapping $\Phi$ : $X \times X_{i} \rightarrow 2^{Z_{i}}$ is called to be $C_{i}(x)$-0-partially diagonally quasiconvex if, for any finite set $\left\{z_{i_{1}}, z_{i_{2}}, \ldots, z_{i_{n}}\right\} \in X_{i}$, and for all $x=\left(x^{i}, x_{i}\right) \in X$ with $x_{i} \in \operatorname{Co}\left\{z_{i_{1}}, z_{i_{2}}, \ldots, z_{i_{n}}\right\}$, there exists some $j$ in $\{1,2, \ldots, n\}$ such that $\Phi\left(x, z_{i_{j}}\right) \not \subset-\operatorname{int} C_{i}(x)$.

It is clear that if $Z_{i}=R$ and $C_{i}(x)=\{r \in R: r \geq 0\}$ for all $x \in X$, and $\Phi_{i}$ is a singlevalued function, then $C_{i}(x)$-0-partially diagonally quasiconvexity of $\Phi_{i}$ reduces to the 0 -partially diagonally quasiconvex (i.e., [14, Definition 3]), furthermore, let $I=\{1\}$, [14, Definition 3] reduces to the $\gamma$-diagonal quasiconvexity in [31,32], here $\gamma=0$.

It is need to recall the following definitions for set-valued mappings in $[6,8]$.

Definition 2.2. Let $E$ and $Z$ be topological spaces, $X \subset E$ a nonempty convex set. Let $C: X \rightarrow 2^{Z}$ be a set-valued mapping with $\operatorname{Int} C(x) \neq \varnothing$ for all $x \in X$ and $\Phi: X \times X \rightarrow 2^{Z}$ be a set-valued mapping. Then $\Phi(x, z)$ is said to be $C(x)$-quasiconvex-like if, for all $x \in X$, $y_{1}, y_{2} \in X$, and $\alpha \in[0,1]$, we have either

$$
\Phi\left(x, \alpha y_{1}+(1-\alpha) y_{2}\right) \subset \Phi\left(x, y_{1}\right)-C(x)
$$

or

$$
\Phi\left(x, \alpha y_{1}+(1-\alpha) y_{2}\right) \subset \Phi\left(x, y_{2}\right)-C(x)
$$

Definition 2.3. Let $X$ and $Y$ be two topological spaces and $T: X \rightarrow 2^{Y}$ be a set-valued mapping.

(1) $T$ is said to be upper semicontinuous if the set $\{x \in X: T(x) \subset V\}$ is open in $X$ for every open subset $V$ of $Y$.

(2) $T$ is said to have open lower sections if the set $T^{-1}(y)=\{x \in X: y \in T(x)\}$ is open in $X$ for each $y \in Y$.

\section{Existence results}

Some existence results of a solution for the (SGSEP) are first be shown as follows.

Theorem 3.1. Let I be an index set and I be countable. For each $i \in I$, let $Z_{i}$ be a real topological vector space, $E_{i}$ and $F_{i}$ be two locally convex Hausdorff topological vector spaces, $X_{i} \subset E_{i}$ be a nonempty, convex and metrizable set and $Y_{i} \subset F_{i}$ be a nonempty, compact, convex and metrizable set, let $C_{i}: X \rightarrow 2^{Z_{i}}$ be a set-valued mapping such that $C_{i}(x)$ is a closed pointed and convex cone with int $C_{i}(x) \neq \varnothing$ for each $x \in X, T_{i}: X \rightarrow 2^{Y_{i}}$ and $\Psi_{i}$ : $X \times Y_{i} \times X_{i} \rightarrow 2^{Z_{i}}$ be set-valued mappings. For each $i \in I$, assume that the following.

(i) $M_{i}=Z_{i} \backslash\left(-\operatorname{int} C_{i}\right): X \rightarrow 2^{Z_{i}}$ is upper semicontinuous.

(ii) For each $y_{i} \in X_{i}, \Psi_{i}\left(x, y_{i}, z_{i}\right)$ is $C_{i}(x)$-0-partially diagonally quasiconvex.

(iii) For all $z_{i} \in X_{i},\left(x, y_{i}\right) \mapsto \Psi_{i}\left(x, y_{i}, z_{i}\right)$ is upper semicontinuous on $X \times Y_{i}$ with compact values. 
(iv) $T_{i}: X \rightarrow 2^{Y_{i}}$ is an upper semicontinous set-valued mapping with nonempty compact values.

(v) For each $i \in I$, there exists a nonempty compact subset $K_{i} \subset X_{i}$ and a compact convex set $B_{i} \subset X_{i}$; let $K=\prod_{i \in I} K_{i} \subset X$ and $B=\prod_{i \in I} B_{i} \subset X$ such that, for each $x \in X \backslash K$, there exists $z_{i}^{*} \in B_{i}$ such that $\Psi_{i}\left(x, y_{i}, z_{i}^{*}\right) \subset-\operatorname{int} C_{i}(x), \forall y_{i} \in T_{i}(x)$.

Then, there exists $\left(\bar{x}, \overline{y_{i}}\right)=\left(\overline{x^{i}}, \overline{x_{i}}, \overline{y_{i}}\right)$ in $K \times Y_{i}$ such that for each $i \in I$,

$$
\overline{x_{i}} \in K_{i}, \quad \overline{y_{i}} \in T_{i}(\bar{x}): \Psi_{i}\left(\bar{x}, \overline{y_{i}}, z_{i}\right) \not \subset-\operatorname{int} C_{i}(\bar{x}), \quad \forall z_{i} \in X_{i} .
$$

Proof

Case 1. For each $i \in I$, the set $X_{i}$ is a compact set.

For each $i \in I$, define a set-valued mapping $P_{i}: X \times Y_{i} \rightarrow 2^{X_{i}}$ by

$$
P_{i}\left(x, y_{i}\right)=\left\{z_{i} \in X_{i}: \Psi_{i}\left(x, y_{i}, z_{i}\right) \subset-\operatorname{int} C_{i}(x)\right\}, \quad \forall\left(x, y_{i}\right) \in X \times Y_{i}
$$

It is need to prove that $x_{i} \notin \operatorname{Co}\left(P_{i}\left(x, y_{i}\right)\right)$ for all $\left(x, y_{i}\right)=\left(x^{i}, x_{i}, y_{i}\right) \in X \times Y_{i}$, where $\operatorname{Co} A$ denotes the convex hull of the set $A$. To see this, suppose, by way of contradiction, that there exist some $i \in I$ and some point $\left(\bar{x}, \overline{y_{i}}\right) \in X \times Y_{i}$ such that $\overline{x_{i}} \in \operatorname{Co}\left(P_{i}\left(\bar{x}, \overline{y_{i}}\right)\right)$. Then there exist finite points $z_{i_{1}}, z_{i_{2}}, \ldots, z_{i_{n}}$ in $X_{i}$, and $\alpha_{j} \geq 0$ with $\sum_{j=1}^{n} \alpha_{j}=1$ such that $\bar{x}=$ $\sum_{j=1}^{n} \alpha_{j} z_{i_{j}}$ and $z_{i_{j}} \in P_{i}\left(\bar{x}, \overline{y_{i}}\right)$ for all $j=1,2, \ldots, n$. That is, $\Psi_{i}\left(\bar{x}, \overline{x_{i}}, z_{i_{j}}\right) \in-\operatorname{int} C_{i}(\bar{x}), j=$ $1,2, \ldots, n$, which contradicts the fact that $\Psi_{i}\left(x, \overline{y_{i}}, z_{i}\right)$ is $C_{i}(x)$-0-partially diagonally quasiconvex.

Now, it is need to prove that the set $P_{i}^{-1}\left(z_{i}\right)=\left\{\left(x, y_{i}\right) \in X \times Y_{i}: \Psi_{i}\left(x, y_{i}, z_{i}\right) \subset\right.$ $\left.-\operatorname{int} C_{i}(x)\right\}$ is open for each $i \in I$ and for each $z_{i} \in X_{i}$. That is, $P_{i}$ has open lower sections on $X \times Y_{i}$. It is only need to prove that $Q_{i}\left(z_{i}\right)=\left\{\left(x, y_{i}\right) \in X \times Y_{i}: \Psi_{i}\left(x, y_{i}, z_{i}\right) \not \subset\right.$ $\left.-\operatorname{int} C_{i}(x)\right\}$ is closed for all $z_{i} \in X_{i}$. In fact, consider a net $\left(x_{t}, y_{i_{t}}\right) \in Q_{i}\left(z_{i}\right)$ such that $\left(x_{t}, y_{i_{t}}\right) \rightarrow\left(x, y_{i}\right) \in X \times Y_{i}$. Since $\left(x_{t}, y_{i_{t}}\right) \in Q_{i}\left(z_{i}\right)$, there exists $u_{t} \in \Psi_{i}\left(x_{t}, y_{i_{t}}, z_{i}\right)$ such that $u_{t} \notin-\operatorname{int} C_{i}\left(x_{t}\right)$. From the upper semicontinuity and compact values of $\Psi_{i}$ on $X \times Y_{i}$ and [29, Proposition 1], it suffices to find a subset $\left\{u_{t_{j}}\right\}$ which converges to some $u \in$ $\Psi_{i}\left(x, y_{i}, z_{i}\right)$, where $u_{t_{j}} \in \Psi_{i}\left(x_{t_{j}}, y_{i_{t_{j}}}, z_{i}\right)$. Since $\left(x_{t_{j}}, y_{i_{t_{j}}}\right) \rightarrow\left(x, y_{i}\right)$, by [8, Proposition 7, page $110]$ and the upper semicontinuity of $M_{i}$, it follows that $u \notin-\operatorname{int} C_{i}(x)$, and hence $\left(x, y_{i}\right)$ $\in Q_{i}\left(z_{i}\right), Q_{i}\left(z_{i}\right)$ is closed.

For each $i \in I$, also define another set-valued mapping $G_{i}: X \times Y_{i} \rightarrow 2^{X_{i}}$ by

$$
G_{i}\left(x, y_{i}\right)=\operatorname{Co}\left(P_{i}\left(x, y_{i}\right)\right), \quad \forall\left(x, y_{i}\right) \in X \times Y_{i}
$$

Let $W_{i}=\left\{\left(x, y_{i}\right) \in X \times Y_{i}: G_{i}\left(x, y_{i}\right) \neq \varnothing\right\}$. Since $P_{i}$ has open lower sections in $X$, and by [30, Lemma 5], we know that $\operatorname{Co}\left(P_{i}\right)$ has open lower sections in $X \times Y_{i}$. Then, for each $z_{i} \in X_{i}, G_{i}^{-1}\left(z_{i}\right)=\left(\operatorname{Co} P_{i}\right)^{-1}\left(z_{i}\right)$ is open, that is, $G_{i}$ also has open lower sections in $X \times Y_{i}$. Hence, $W_{i}=\cup_{z_{i} \in X_{i}} G_{i}^{-1}\left(z_{i}\right)$ is an open set in $X \times Y_{i}$. Then, the set-valued mapping $\left.G_{i}\right|_{W_{i}}$ : $W_{i} \rightarrow 2^{X_{i}}$ has open lower sections in $W_{i}$, and for all $\left(x, y_{i}\right) \in W_{i}, G_{i}\left(x, y_{i}\right)$ is nonempty and convex. Also, since $X \times Y_{i}$ is metrizable space [20, page 50], $W_{i}$ is paracompact [24, page 831]. Hence, by [30, Lemma 6], there is a continuous function $s_{i}: W_{i} \rightarrow X_{i}$ such that 
6 The system of generalized set-valued equilibrium problems

$s_{i}\left(x, y_{i}\right) \in G_{i}\left(x, y_{i}\right)$ for all $\left(x, y_{i}\right) \in W_{i}$. Define $H_{i}: X \times Y_{i} \rightarrow 2^{X_{i}}$ by

$$
H_{i}\left(x, y_{i}\right)= \begin{cases}\left\{s_{i}\left(x, y_{i}\right)\right\} & \text { if }\left(x, y_{i}\right) \in W_{i} \\ X_{i} & \text { if }\left(x, y_{i}\right) \notin W_{i}\end{cases}
$$

It is easy to prove that $H_{i}$ is upper semicontinuous.

Now define a set-valued mapping $\Gamma: X \times Y \rightarrow 2^{X \times Y}$ by $\Gamma(x, y)=\left(\prod_{i \in I} H_{i}\left(x, y_{i}\right)\right.$, $\left.\prod_{i \in I} T_{i}(x)\right)$, for each $(x, y) \in X \times Y$. By [17, Lemma 3], $\Gamma$ is upper semicontinuous. Since for each $(x, y) \in X \times Y, H(x, y)$ is convex, closed, and nonempty, by [17, Theorem 1], there is $(\bar{x}, \bar{y}) \in X \times Y$ such that $(\bar{x}, \bar{y}) \in H(\bar{x}, \bar{y})$. Note that for each $i \in I,\left(\bar{x}, \overline{y_{i}}\right) \notin W_{i}$. Otherwise, there is some $i \in I$ such that $\left(\bar{x}, \overline{y_{i}}\right) \in W_{i}$, then $\overline{x_{i}}=s_{i}\left(\bar{x}, \overline{y_{i}}\right) \in \operatorname{Co}\left(P_{i}\left(\bar{x}, \overline{y_{i}}\right)\right)$, which contradicts $x_{i} \notin \operatorname{Co}\left(P_{i}\left(x, y_{i}\right)\right)$ for all $\left(x, y_{i}\right)=\left(x^{i}, x_{i}, y_{i}\right) \in X \times Y_{i}$. Thus $\overline{x_{i}} \in X_{i}, \overline{y_{i}} \in$ $T_{i}(\bar{x})$ and $G_{i}\left(\bar{x}, \overline{y_{i}}\right)=\varnothing$ for each $i \in I$. That is, $\bar{x} \in X, \overline{y_{i}} \in T_{i}(\bar{x})$ and $\operatorname{Co}\left(P_{i}\left(\bar{x}, \overline{y_{i}}\right)\right)=\varnothing$ for each $i \in I$, which implies $\bar{x} \in X, \overline{y_{i}} \in T_{i}(\bar{x})$ and $P_{i}\left(\bar{x}, \overline{y_{i}}\right)=\varnothing$ for each $i \in I$. Consequently, there exists $\left(\bar{x}, \overline{y_{i}}\right)$ in $X \times Y_{i}$ such that for each $i \in I, \bar{x} \in X$ and $\overline{y_{i}} \in T_{i}(\bar{x})$ : $\Psi_{i}\left(\bar{x}, \overline{y_{i}}, z_{i}\right) \not \subset-\operatorname{int} C_{i}(\bar{x}), \forall z_{i} \in X_{i}$.

Case 2. $X_{i}$ is not a compact set.

For each $i \in I$, let $\left\{z_{i_{1}}, \ldots, z_{i_{k}}\right\}$ be a finite subset of $X_{i}$. Let $\Lambda_{i}=\operatorname{Co}\left(B_{i} \cup\left\{z_{i_{1}}, \ldots, z_{i_{k}}\right\}\right)$. Then, for each $i \in I, \Lambda_{i}$ is compact and convex. By Case 1, there exists $\bar{x} \in \Lambda=\prod_{i \in I} \Lambda_{i}$ and $\overline{y_{i}} \in T_{i}(\bar{x})$ for each $i \in I$ such that, for each $i \in I, \Psi_{i}\left(\bar{x}, \overline{y_{i}}, z_{i}\right) \not \subset-\operatorname{int} C_{i}(\bar{x})$, for all $z_{i} \in \Lambda_{i}$. From $B \subset \Lambda$ and assumption (v) it follows that $\bar{x} \in K$. In particular, we have, $(\bar{x}, \bar{y}) \in K \times Y$ such that, for each $i \in I, \Psi_{i}\left(\bar{x}, \overline{y_{i}}, z_{i_{j}}\right) \not \subset-\operatorname{int} C_{i}(\bar{x})$, for all $j=1,2, \ldots, k$. By (vi) and [8, Proposition 7, page 110], the set $\left\{\left(x, y_{i}\right) \in K \times Y_{i}: y_{i} \in T_{i}(x)\right\}$ is closed in $K \times Y_{i}$. Hence, for each $i \in I$ and for all $z_{i} \in X_{i}, \Delta\left(z_{i}\right)=\left\{\left(x, y_{i}\right) \in K \times Y_{i}: F_{i}\left(x, y_{i}, z_{i}\right) \not \subset\right.$ $\left.-\operatorname{int} C_{i}(x), y_{i} \in T_{i}(x)\right\}=Q_{i}\left(z_{i}\right) \cap\left\{\left(x, y_{i}\right) \in K \times Y_{i}: y_{i} \in T_{i}(x)\right\}$ is closed in $K \times Y_{i}$. Since every finite subfamily of closed sets $\Delta\left(z_{i}\right)$ in compact set $K \times Y_{i}$ has a nonempty intersection, for each $i \in I, \cap_{z_{i} \in X_{i}} \Delta\left(z_{i}\right) \neq \varnothing$. Thus, there exists $(\bar{x}, \bar{y}) \in K \times Y$ such that, for each $i \in I, \overline{x_{i}} \in K_{i}, \overline{y_{i}} \in T_{i}(\bar{x})$, such that $\Psi_{i}\left(\bar{x}, \overline{y_{i}}, z_{i}\right) \not \subset-\operatorname{int} C_{i}(\bar{x})$, for all $z_{i} \in X_{i}$. This completes the proof.

Theorem 3.2. If we replace, in Theorem 3.1, condition (ii) by the following conditions.

(ii(a)) For all $x=\left(x^{i}, x_{i}\right) \in X$, for all $y_{i} \in Y_{i}, \Psi_{i}\left(x, y_{i}, x_{i}\right) \not \subset-\operatorname{int} C_{i}(x)$.

(ii(b)) For each $\left(x, y_{i}\right) \in X \times Y_{i}$, the set $P_{i}\left(x, y_{i}\right)=\left\{z_{i} \in X_{i}: \Psi_{i}\left(x, y_{i}, z_{i}\right) \subset-\operatorname{int} C_{i}(x)\right\}$ is a convex set.

Then, the conclusion of Theorem 3.1 still holds.

Proof. By Theorem 3.1, it is only need to prove that $\Psi_{i}\left(x, y_{i}, z_{i}\right)$ is $C_{i}(x)$-0-partially diagonally quasiconvex for each $i \in I$ and for all $y_{i} \in X_{i}$. If not, then there exist some $i \in I$ and $y_{i} \in X_{i}$, some finite set $\left\{z_{i_{1}}, z_{i_{2}}, \ldots, z_{i_{n}}\right\}$ in $X_{i}$, and some point $x=\left(x^{i}, x_{i}\right) \in$ $X$ with $x_{i} \in \operatorname{Co}\left\{z_{i_{1}}, z_{i_{2}}, \ldots, z_{i_{n}}\right\}$. Then, for each $j=1,2, \ldots, n, \Psi_{i}\left(x, y_{i}, z_{i_{j}}\right) \subset-\operatorname{int} C_{i}(x)$. Since $P_{i}\left(x, y_{i}\right)=\left\{z_{i} \in X_{i}: \Psi_{i}\left(x, y_{i}, z_{i}\right) \subset-\operatorname{int} C_{i}(x)\right\}$ is a convex set, $x_{i} \in P_{i}\left(x, y_{i}\right)$, that is, $\Psi_{i}\left(x, y_{i}, x_{i}\right) \subset-\operatorname{int} C_{i}(x)$, which contradicts to the condition (ii). This completes the proof. 
Theorem 3.3. If we replace, in Theorem 3.2, condition (ii(b)) by the following condition. (ii(c)) For each $y_{i} \in Y_{i}, \Psi_{i}\left(x, y_{i}, z_{i}\right)$ is $C_{i}(x)$-convex-like.

Then, the conclusion of Theorem 3.2 still holds.

Proof. For each $i \in I$, define a set-valued mapping $P_{i}: X \times Y_{i} \rightarrow 2^{X_{i}}$ by $P_{i}\left(x, y_{i}\right)=\left\{z_{i} \in\right.$ $\left.X_{i}: \Psi_{i}\left(x, y_{i}, z_{i}\right) \subset-\operatorname{int} C_{i}(x)\right\}, \forall\left(x, y_{i}\right) \in X \times Y_{i}$. It is easy to prove that for each $i \in I$ and for each $\left(x, y_{i}\right) \in X \times Y_{i}$, the set $P_{i}\left(x, y_{i}\right)$ is a convex set. By Theorem 3.2, the conclusion of Theorem 3.3 holds. This completes the proof.

Then, some existence results for the special cases of the (SGSEP) will be considered.

Corollary 3.4. Let $I$ be an index set and $I$ be countable. For each $i \in I$, let $Z_{i}$ be a real topological vector space, $E_{i}$ be a locally convex Hausdorff topological vector space, $X_{i} \subset E_{i}$ be a nonempty, convex and metrizable set, let $C_{i}: X \rightarrow 2^{Z_{i}}$ be a set-valued mapping such that $C_{i}(x)$ is a closed pointed and convex cone with int $C_{i}(x) \neq \varnothing$ for each $x \in X$, let $\Phi_{i}$ : $X \times X_{i} \rightarrow 2^{Z_{i}}$ be a set-valued mapping. For each $i \in I$, assume that the following.

(i) The set-valued mapping $M_{i}=Z_{i} \backslash\left(-\operatorname{int} C_{i}\right): X \rightarrow 2^{Z_{i}}$ is upper semicontinuous.

(ii) $\Phi_{i}\left(x, z_{i}\right)$ is $C_{i}(x)$-0-partially diagonally quasiconvex.

(iii) For all $z_{i} \in X_{i}$, the map $x \mapsto \Phi_{i}\left(x, z_{i}\right)$ is upper semicontinuous on $X$ with compact values.

(vi) There exists a nonempty compact subset $K_{i} \subset X_{i}$ and a compact convex set $B_{i} \subset X_{i}$; let $K=\prod_{i \in I} K_{i} \subset X$ and $B=\prod_{i \in I} B_{i} \subset X$ such that, for each $x \in X \backslash K$, there exists $z_{i}^{*} \in B_{i}$ such that $\Phi_{i}\left(x, z_{i}^{*}\right) \subset-\operatorname{int} C_{i}(x)$.

Then, there exists $\bar{x}$ in $K$ such that for each $i \in I, \Phi_{i}\left(\bar{x}, z_{i}\right) \not \subset-\operatorname{int} C_{i}(\bar{x}), \forall z_{i} \in X_{i}$.

Proof. For each $i \in I$, Let $Y_{i}=\left\{\overline{y_{i}}\right\}$ and define a set-valued mapping $T_{i}: X \rightarrow 2^{Y_{i}}$ as $T_{i}(x)=\left\{\overline{y_{i}}\right\}$ for all $x \in X$ and define another set-valued mapping $\Psi_{i}: X \times Y_{i} \times X_{i}$ as $\Psi_{i}\left(x, \overline{y_{i}}, z_{i}\right)=\Phi_{i}\left(x, z_{i}\right), \forall\left(x, \overline{y_{i}}, z_{i}\right) \in X \times Y_{i} \times X_{i}$. It is easy to see that all conditions of Theorem 3.1 are satisfied. Then the conclusion of Corollary 3.4 follows from Theorem 3.1. This completes the proof.

Corollary 3.5. If we replace, in Corollary 3.4, condition (ii) by the following conditions.

(a) For each $x \in X,\left\{z_{i} \in X_{i}: \Phi_{i}\left(x, z_{i}\right) \subset-\operatorname{int} C_{i}(x)\right\}$ is a convex set (or $\Phi\left(x, z_{i}\right)$ is $C_{i}(x)$ convex-like).

(b) For all $x=\left(x^{i}, x_{i}\right) \in X, \Phi_{i}\left(x, x_{i}\right) \not \subset C_{i}(x)$.

Then, the conclusion of Corollary 3.4 still holds.

Remark 3.6. Theorems 3.1-3.3, Corollaries 3.4 and 3.5 improve and generalize [4, Theorems 2 and 3], [3, Theorems 2.1 and 2.2] with additional conditions of the metrizability of $X_{i}$.

Remark 3.7. By the results in this paper, it is easy to obtain the existence results for the other special cases of the (SGSEP), and they are omitted here.

\section{Acknowledgments}

The author would like to express his thanks to the referees for helpful suggestions. This research was partially supported by the National Natural Science Foundation of China 
8 The system of generalized set-valued equilibrium problems

(Grant no. 10171118) and Education Committee Project Research Foundation of Chongqing (Grant no. 030801) and the Science Committee Project Research Foundation of Chongqing (Grant no. 8409).

\section{References}

[1] E. Allevi, A. Gnudi, and I. V. Konnov, Generalized vector variational inequalities over product sets, Nonlinear Analysis 47 (2001), no. 1, 573-582.

[2] Q. H. Ansari, I. V. Konnov, and J.-C. Yao, On generalized vector equilibrium problems, Nonlinear Analysis 47 (2001), no. 1, 543-554.

[3] Q. H. Ansari, S. Schaible, and J.-C. Yao, System of vector equilibrium problems and its applications, Journal of Optimization Theory and Applications 107 (2000), no. 3, 547-557.

[4] - The system of generalized vector equilibrium problems with applications, Journal of Global Optimization 22 (2002), no. 1-4, 3-16.

[5] Q. H. Ansari and J.-C. Yao, A fixed point theorem and its applications to a system of variational inequalities, Bulletin of the Australian Mathematical Society 59 (1999), no. 3, 433-442.

[6] __ An existence result for the generalized vector equilibrium problem, Applied Mathematics Letters 12 (1999), no. 8, 53-56.

[7] __ Systems of generalized variational inequalities and their applications, Applicable Analysis 76 (2000), no. 3-4, 203-217.

[8] J.-P. Aubin and I. Ekeland, Applied Nonlinear Analysis, Pure and Applied Mathematics (New York), John Wiley \& Sons, New York, 1984.

[9] M. Bianchi, Pseudo P-monotone operators and variational inequalities, Report No. 6, Istituto di Econometria e Matematica per le Decisioni Economiche, Universita Cattolica del Sacro Cuore, Milan, 1993.

[10] M. Bianchi, N. Hadjisavvas, and S. Schaible, Vector equilibrium problems with generalized monotone bifunctions, Journal of Optimization Theory and Applications 92 (1997), no. 3, 527-542.

[11] M. Bianchi and S. Schaible, Generalized monotone bifunctions and equilibrium problems, Journal of Optimization Theory and Applications 90 (1996), no. 1, 31-43.

[12] E. Blum and W. Oettli, From optimization and variational inequalities to equilibrium problems, The Mathematics Student 63 (1994), no. 1-4, 123-145.

[13] O. Chadli, Y. Chiang, and S. Huang, Topological pseudomonotonicity and vector equilibrium problems, Journal of Mathematical Analysis and Applications 270 (2002), no. 2, 435-450.

[14] G. Y. Chen and H. Yu, Existence of solutions to a random equilibrium system, Journal of Systems Science and Mathematical Sciences 22 (2002), no. 3, 278-284 (Chinese).

[15] G. Cohen and F. Chaplais, Nested monotony for variational inequalities over product of spaces and convergence of iterative algorithms, Journal of Optimization Theory and Applications 59 (1988), no. 3, 369-390.

[16] P. Deguire, K. K. Tan, and G. X.-Z. Yuan, The study of maximal elements, fixed points for $L_{s^{-}}$ majorized mappings and their applications to minimax and variational inequalities in product topological spaces, Nonlinear Analysis 37 (1999), no. 7, 933-951.

[17] K. Fan, Fixed-point and minimax theorems in locally convex topological linear spaces, Proceedings of the National Academy of Sciences of the United States of America 38 (1952), 121-126.

[18] J.-Y. Fu and A.-H. Wan, Generalized vector equilibrium problems with set-valued mappings, Mathematical Methods of Operations Research 56 (2002), no. 2, 259-268.

[19] N. Hadjisavvas and S. Schaible, From scalar to vector equilibrium problems in the quasimonotone case, Journal of Optimization Theory and Applications 96 (1998), no. 2, 297-309.

[20] J. L. Kelley and I. Namioka, Linear topological spaces, The University Series in Higher Mathematics, D. Van Nostrand, New Jersey, 1963. 
[21] I. V. Konnov, Relatively monotone variational inequalities over product sets, Operations Research Letters 28 (2001), no. 1, 21-26.

[22] I. V. Konnov and J.-C. Yao, Existence of solutions for generalized vector equilibrium problems, Journal of Mathematical Analysis and Applications 233 (1999), no. 1, 328-335.

[23] L. J. Lin, Z. T. Yu, and G. Kassay, Existence of equilibria for multivalued mappings and its application to vectorial equilibria, Journal of Optimization Theory and Applications 114 (2002), no. 1, 189-208.

[24] E. Michael, A note on paracompact spaces, Proceedings of the American Mathematical Society 4 (1953), 831-838.

[25] W. Oettli, A remark on vector-valued equilibria and generalized monotonicity, Acta Mathematica Vietnamica 22 (1997), no. 1, 213-221.

[26] W. Oettli and D. Schläger, Existence of equilibria for monotone multivalued mappings, Mathematical Methods of Operations Research 48 (1998), no. 2, 219-228.

[27] J.-S. Pang, Asymmetric variational inequality problems over product sets: applications and iterative methods, Mathematical Programming 31 (1985), no. 2, 206-219.

[28] J. W. Peng, Equilibrium problems for W-spaces, Mathematica Applicata (Wuhan) 12 (1999), no. 3, 81-87 (Chinese).

[29] C. H. Su and V. M. Sehgal, Some fixed point theorems for condensing multifunctions in locally convex spaces, Proceedings of the American Mathematical Society 50 (1975), 150-154.

[30] G. Q. Tian and J. Zhou, Quasi-variational inequalities without the concavity assumption, Journal of Mathematical Analysis and Applications 172 (1993), no. 1, 289-299.

[31] S. S. Zhang, Variational inequalities and complementarity problem theory with applications, Shanghai Science and Technology, Shanghai, 1991.

[32] J. Zhou and G. Chen, Diagonal convexity conditions for problems in convex analysis and quasivariational inequalities, Journal of Mathematical Analysis and Applications 132 (1988), no. 1, 213-225.

Jian-Wen Peng: College of Mathematics and Computer Science, Chongqing Normal University, Chongqing 400047, China

E-mail address: jwpeng6@yahoo.com.cn 